
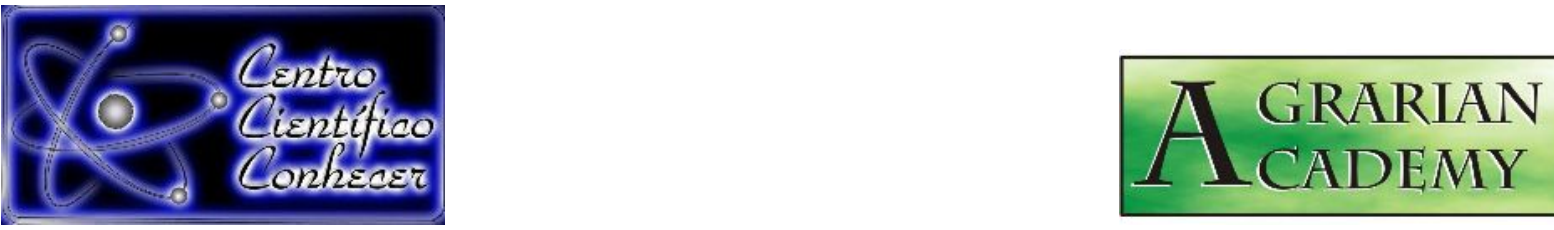

\title{
DINÂMICA DA TEMPERATURA SUPERFÍCIE DO PERÍMETRO URBANO DE ALTA FLORESTA, MATO GROSSO
}

\footnotetext{
Charles Caioni

Doutor em Ecologia e Conservação pela Universidade Estado de Mato Grosso, Alta

Floresta/MT, Brasil, (charlescaione@hotmail.com)

Recebido em: 15/06/2021 - Aprovado em: 15/07/2021 - Publicado em: 30/07/2021

DOI: 10.18677/Agrarian_Academy_2021A3
}

\begin{abstract}
RESUMO
Mudanças no uso da terra podem alterar o balanço de energético. Nos centros urbanos o aumento de áreas pavimentadas tem ocasionado o aumento da Temperatura Superficial (TS), surgindo assim o fenômeno ilhas de calor. Esse microclima urbano pode ocasionar tantos danos econômicos quanto à saúde da população. Nessa conjectura, objetivou-se estudar a dinâmica (de 2013 a 2020) da temperatura da superfície do perímetro urbano no município de alta Floresta, Mato Grosso. Os mapas temáticos foram obtidos a partir de duas imagens (datadas em 07/07/2013 e 12/09/2020) dos sensores Operational Land Imager (bandas 4, 5 e 6) e Thermal Infrared (banda 10), ambos a bordo do satélite Landsat 8. Constataram-se mudanças no uso e cobertura da terra entre 2013 e 2020. A classe cobertura vegetal reduziu $6,64 \mathrm{~km}^{2}$ ao mesmo tempo em que as áreas pavimentadas aumentaram $5,75 \mathrm{Km}^{2}$. Em resposta a essas mudanças a TS aumentou $\sim 0,8^{\circ} \mathrm{C}$. Embora os ambientes com cobertura vegetal tenham demonstrado contribuir para a redução da TS na cidade, pode-se observar que a TS também tem aumentado nesses ambientes $\left(0,43^{\circ} \mathrm{C}\right)$, possivelmente devido ao aumento das áreas pavimentadas que tende a expressar elevadas temperaturas de superfície. Nessa perspectiva, recomenda-se o desenvolvimento de outros estudos, tendo em vista os serviços ecossistêmicos prestados pelos remanescentes florestais.
\end{abstract}

PALAVRAS-CHAVE: Crescimento urbano, ilhas de calor, uso da terra.

\section{SURFACE TEMPERATURE DYNAMICS FROM ALTA FLORESTA URBAN PERIMETER, MATO GROSSO}

\begin{abstract}
Changes in land use can alter the energy balance. In urban centers, the increase in paved areas has caused an increase in the Surface Temperature (TS), thus giving rise to the phenomenon of heat islands. This urban microclimate can cause as much economic damage as the health of the population. In this conjecture, the objective was to study the dynamics (from 2013 to 2020) of the surface temperature of the
\end{abstract}


urban perimeter in the municipality of Alta Floresta, Mato Grosso. The thematic maps were obtained from two images (dated 07/07/2013 and 12/09/2020) of the Operational Land Imager (bands 4, 5 and 6) and Thermal Infrared (band 10) sensors, both on board the Landsat satellite 8 . Changes were noted in land use and land cover between 2013 and 2020 . The vegetation cover class reduced $6.64 \mathrm{~km}^{2}$ while paved areas increased $5.75 \mathrm{~km}^{2}$. In response to these changes the TS increased $\sim 0.8^{\circ} \mathrm{C}$. Although environments with vegetation cover have been shown to contribute to the reduction of TS in the city, it can be observed that TS has also increased in these environments $\left(0.43^{\circ} \mathrm{C}\right)$, possibly due to the increase in paved areas, which tends to express high temperatures of surface. From this perspective, the development of other studies is recommended, considering the ecosystem services provided by forest remnants.

KEYWORDS: Heat islands, land use, urban growth.

\section{INTRODUÇÃO}

Mudanças no uso e ocupação da terra em áreas próximas ou no interior dos centros urbanos podem ocasionar significativas alterações nos componentes do balanço de energia (BEZERRA et al., 2018; CAIONI et al., 2020). Entre esses componentes a temperatura da superfície (TS) é uma das mais afetadas, isso porque grande parte da energia que chega sobre a copa da floresta ou de qualquer outro tipo de vegetação presente dentro do perímetro urbano, acaba sendo convertida em calor latente via evapotranspiração. Assim, quando essa vegetação é removida ou substituída, grande parte dessa energia que antes se tornava calor latente acaba tornando-se calor sensível (SABINO et al., 2019), onde sob tais condições a TS também aumenta, surgindo o que é conhecido como ilhas de calor (BARBOZA et al., 2020).

As ilhas urbanas são fenômenos que podem ocasionar danos tanto econômicos, quanto a saúde (PORANGABA; AMORIM, 2019). Por exemplo, no norte de Mato Grosso grande parte da produção hortícola vem de locais urbanos e periurbanos, assim, o aumento exacerbado da TS e consequentemente da temperatura do ar pode restringir o plantio a apenas espécies mais resistentes a altas temperaturas. $O$ aumento da TS também pode contribuir para a elevação da evapotranspiração, podendo assim ocorrer chuvas mais intensas, que por sua vez, agravarão ainda mais os problemas de alagamento dos bairros com maior declividade. Com relação à saúde, o aumento da TS pode contribuir para o aumento de doenças disseminadas por vetores como dengue, zika e febre chicungunya.

Embora nos últimos anos o número de estudos voltados para análise do surgimento de ilhas de calor tenha aumentado, ainda conhece-se pouco sobre esse fenômeno em regiões tropicais, como por exemplo, na Amazônica. Isso porque grande parte dos trabalhos de campo sobre ilha de calor estão baseados em uma amostragem de curto espaço de tempo, devido ao custo de manutenção dos equipamentos ou até mesmo, devido a atos de vandalismo limitarem os experimentos. Nesta conjectura, o sensoriamento remoto (SR) pode ser considerado uma importante ferramenta na tomada de decisões (ARAUJO et al., 2017; CAIONI et al., 2017, PESSI et al., 2019), visto que permite de forma acurada obter diversos dados climáticos (PORANGABA; AMORIM, 2019).

Em meio a esse contexto encontra-se o perímetro urbano do município de Alta Floresta, que ao longo das quatro últimas décadas tem apresentado uma 
consistente mudança em sua composição, com áreas de florestas dando lugar a pastagens e posteriormente casas, ruas, calçadas e demais infraestruturas. Nessa perspectiva, objetivou-se estudar a dinâmica (de 2013 a 2020) da temperatura da superfície do perímetro urbano no município de alta Floresta, Mato Grosso.

\section{Área de estudo}

\section{MATERIAL E MÉTODOS}

O estudo compreendeu o perímetro urbano do município de Alta Floresta, localizado entre as coordenadas de $56^{\circ} 30^{\prime}$ a $57^{\circ} 00^{\prime}$ de longitude $W$ e $9^{\circ} 00^{\prime}$ a $11^{\circ} 00^{\prime}$ de latitude $S$ (Figura 1). Com uma população estimada em 51.959 habitantes, o IDH é de 0,714 (IBGE, 2020). A região possui clima do tipo Am (monçonico), com nítida estação seca (de dezembro a março) e chuvosa (de junho a setembro). Com chuvas próximas a $2200 \mathrm{~mm}$ (CAIONI et al., 2014), a temperatura anual fica em torno dos $25^{\circ} \mathrm{C}$. A vegetal local é característica da transição Cerrado-Amazônia.

FIGURA 1. Localização do perímetro urbano de Alta Florestal, Mato Grosso.

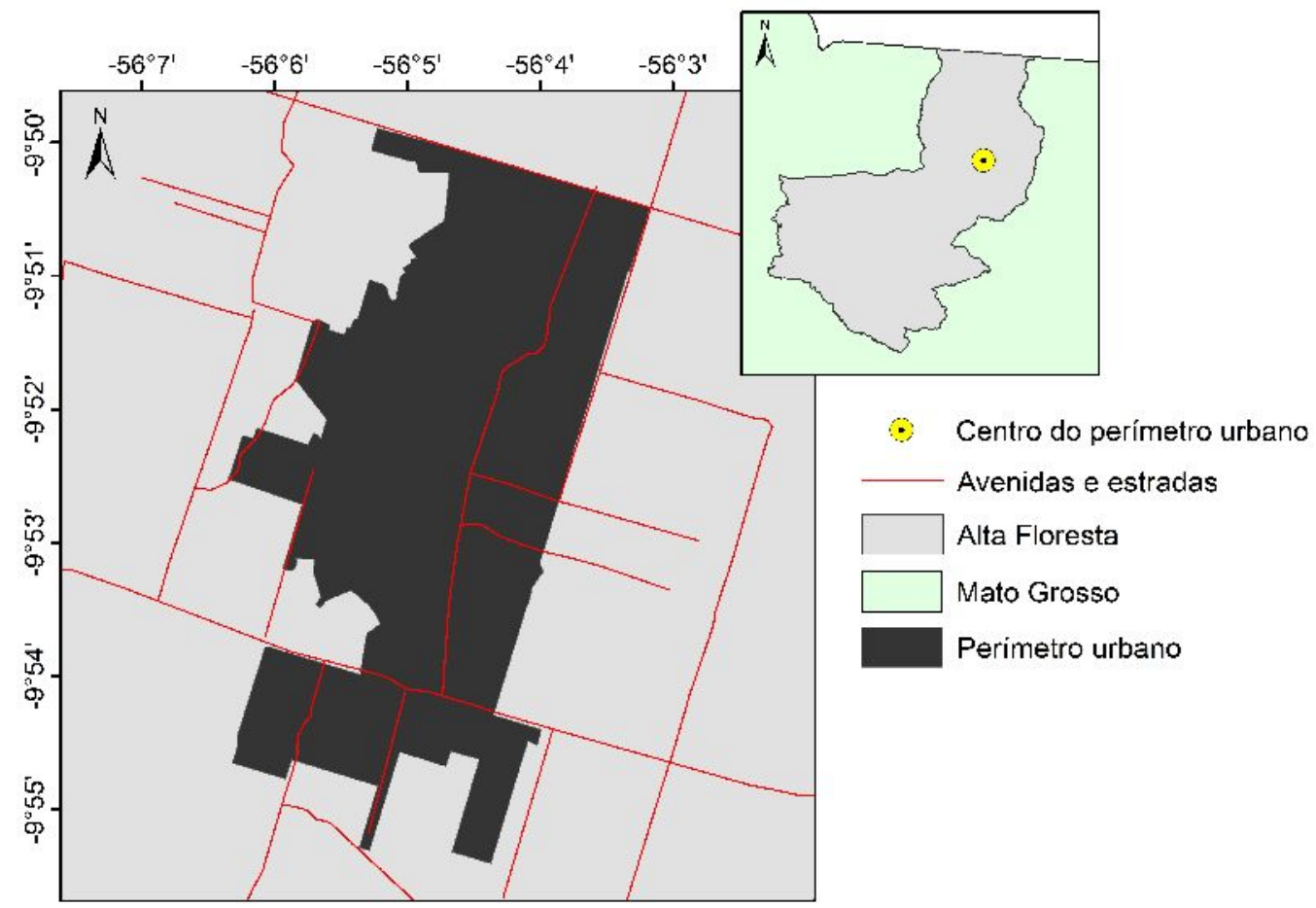

Fonte: próprio autor.

\section{Procedimentos metodológicos}

Para a construção dos mapas de temperatura da superfície e uso da terra foram utilizadas duas imagens dos sensores Operational Land Imager (OLI) e Thermal Infrared a bordo do satélite Landsat 8 (datadas em 07/07/2013 e 12/09/2020), ambas pertencentes a órbita/ponto 227/67. 
Uso e ocupação da terra

Para a confecção dos mapas de uso da terra foi aplicado nas bandas 4,5 e 6 (resolução espacial: 30 metros), os procedimentos de recorte, segmentação e classificação no software SPRING, versão 4.3. (CÂMARA et al., 1996).

Durante a classificação definiu-se as seguintes classes:

- Área pavimentada: Foram consideradas todas as áreas desprovidas de vegetação, como por exemplo, locais pavimentados o com solo exposto;

- Cobertura vegetal: foram consideradas todas as formas de vegetação, como por exemplo, formações florestais e pastagem;

- Massa d'água: Áreas ocupadas com água livre de vegetação, englobando os rios e lagos.

Após a classificação foi realizada a validação do modelo por meio de visitas a campo, para somente então, quantificar cada classe no software $R$ versão 3.0.2 ( $R$ CORE TEAM, 2013).

Temperatura da superfície TS

Por meio do software R versão 3.0.2 (com o auxílio do pacote raster) (HIJMANS, 2014) foram realizados os procedimentos de recorte e conversão dos valores de cinza em radiância na banda 10 de cada cena utilizada no presente estudo (Equação 1).

Sendo:

$$
L \lambda=M L^{*} \text { Qcal }+A L
$$

$L \lambda=$ Radiância Espectral do sensor de abertura em Watts/ $\left(\mathrm{m}^{2} \mathrm{sr} \mathrm{m}\right)$;

$\mathrm{ML}=$ Fator multiplicativo de redimensionamento da banda $10=3.3420 \mathrm{E}-04$;

$\mathrm{AL}=$ Fator de redimensionamento aditivo específico da banda $10=0.10000$;

Qcal= Valor quantizado calibrado pelo pixel em DN = Imagem banda 10.

Após a aquisição dos valores de radiância aplicou-se o algoritmo 2, que permitiu a obtenção dos diferentes níveis de temperatura da superfície.

$$
\mathrm{T}=\frac{\mathrm{K} 2}{\operatorname{in}\left(\frac{\mathrm{K} 1}{\mathrm{~L}}+1\right)}
$$

Onde:

$\mathrm{T}$ : Temperatura efetiva em Kelvin (K);

K2: Constante de calibração $2=1.321 .08(\mathrm{~K})$;

K1: Constante de calibração $1=774.89(\mathrm{~K})$;

$\mathrm{L} \lambda$ : Radiância espectral em Watts $/\left(\mathrm{m}^{2} \mathrm{sr} \mathrm{m}\right)$.

Os valores térmicos foram convertidos de Kelvin para graus Celsius $\left({ }^{\circ} \mathrm{C}\right)$ a partir da equação 3 .

Sendo:

$$
\mathrm{TC}=\mathrm{Tk}-273,15
$$

Tc: Temperatura em Celsius; Tk: Temperatura em Kelvin. terra

Obtenção da temperatura superficial (TS) para os diferentes usos e coberturas da

Com a função STACK do pacote Raster (HIJMANS et al., 2014) foi possível a obtenção da média geral da temperatura da superfície para os anos de 2013 e 2020, bem como a variação dentro de cada classe de uso/cobertura da terra. 


\section{RESULTADOS E DISCUSSÃO}

Entre 2013 e 2020 houve uma considerável redução da cobertura vegetal $(6,64$ $\mathrm{km}^{2}$ ) ao mesmo tempo em que as áreas pavimentadas aumentaram $\left(5,75 \mathrm{Km}^{2}\right)$ (Figura 1, Tabela 1). Este resultado está relacionado as áreas com pastagem terem dado lugar a loteamentos para a construção de casas. Isso fica perceptível ao analisar a figura 2, onde é possível constatar que a redução da cobertura vegetal ocorreu principalmente nas áreas periféricas. Estudos (CAIONI et al. 2014) têm demonstrando que o processo de crescimento da área urbana de Alta Floresta é contínuo, tendo em vista que ainda há locais propícios ao surgimento de novos bairros ou aumento dos já existentes.

FIGURA 2. Dinâmica do uso da terra no perímetro urbano de Alta Florestal, Mato Grosso.

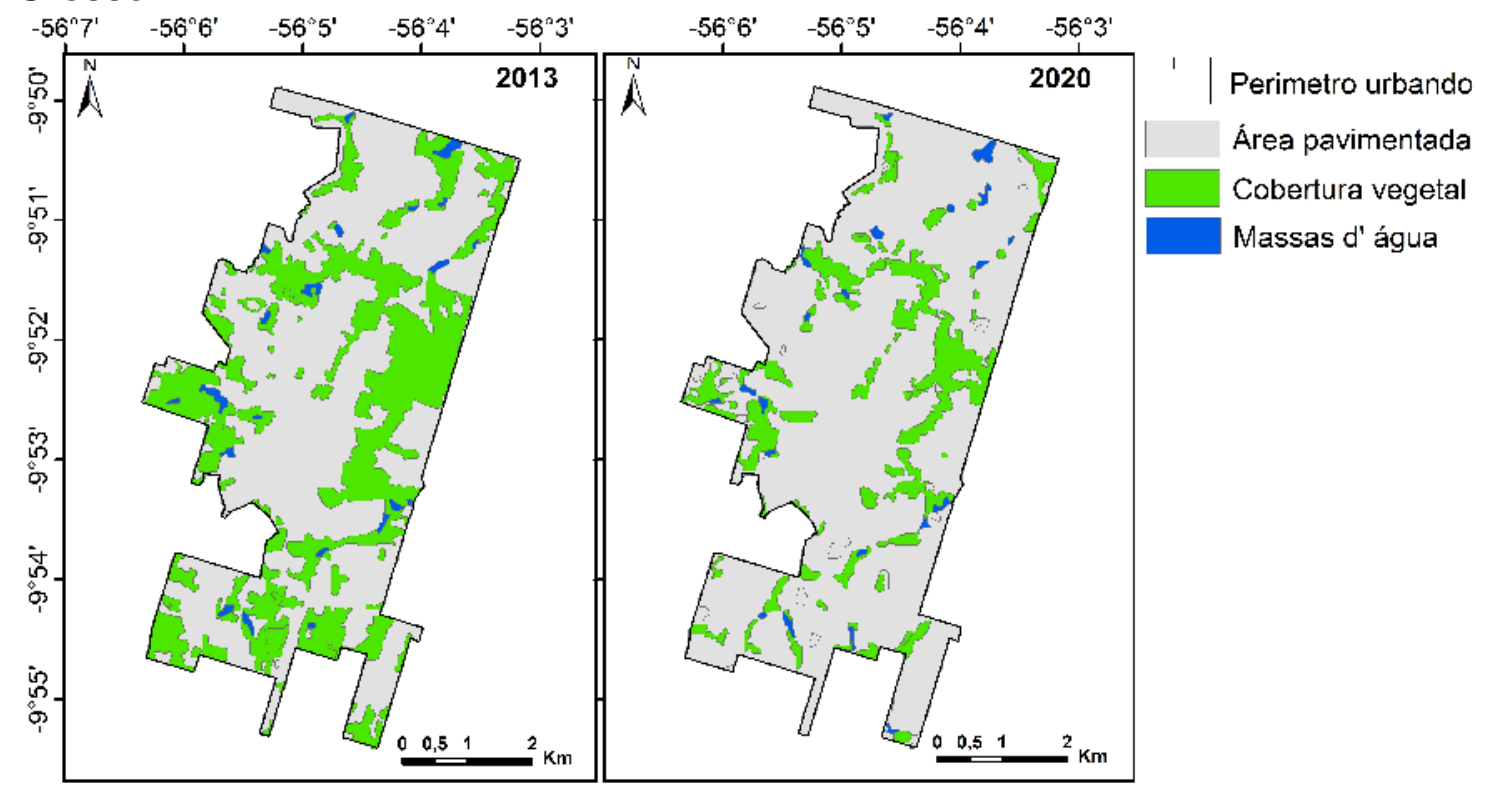

Fonte: próprio autor.

TABELA 1. Uso e cobertura da terra em 2013 e 2020

\begin{tabular}{|c|c|c|}
\hline Área pavimentada & $18,32(62,21)$ & $24,07(81,73)$ \\
\hline Cobertura vegetal & $10,41(35,35)$ & $4,77(16,20)$ \\
\hline Massa d'água & $0,72(2,44)$ & $0,61(2,07)$ \\
\hline TOTAL & 29,45 & 29,45 \\
\hline
\end{tabular}

É possível averiguar também que grande parte da cobertura vegetal remanescente é constituída por ambientes florestais localizados próximos a córregos, lagos e demais hidrografias que percorrem o perímetro urbano. Esses ambientes existem por causa da lei n. 12.651/2012, que delimita a proteção integral de 30 e 50 metros para cursos hídricos e nascentes, respectivamente. A presença de cobertura florestal é essencial, visto que permite não apenas a proteção à ambientes hídricos, mas também o conforto térmico a população. 
Assim como esperado não foram evidenciadas grandes mudanças na classe massa d'agua. Apenas uma redução de $0,11 \mathrm{~km}^{2}$, que pode ser decorrente de um possível erro na classificação do solo, visto que nunca é possível alcançar $100 \%$ de precisão no processo de classificação.

A temperatura da superfície variou ao longo de todo perímetro urbano (figura 3), tanto em 2013 quanto em 2020. Em 2013 as maiores temperaturas ocorreram nas áreas centrais, enquanto em 2020 nas áreas mais periféricas. Este resultado pode estar relacionado aos novos loteamentos ainda possuírem baixa presença de árvores em frente aos lotes criados. Outro fator que pode ter contribuído é a presença de um grande remanescente florestal próximo ao centro da cidade que contribui para a amenização dos altos valores térmicos (PORANGABA et al., 2017; AMORIM, 2019; SILVA et al., 2020).

FIGURA 3. Dinâmica da temperatura da superfície no perímetro urbano de Alta Florestal, Mato Grosso

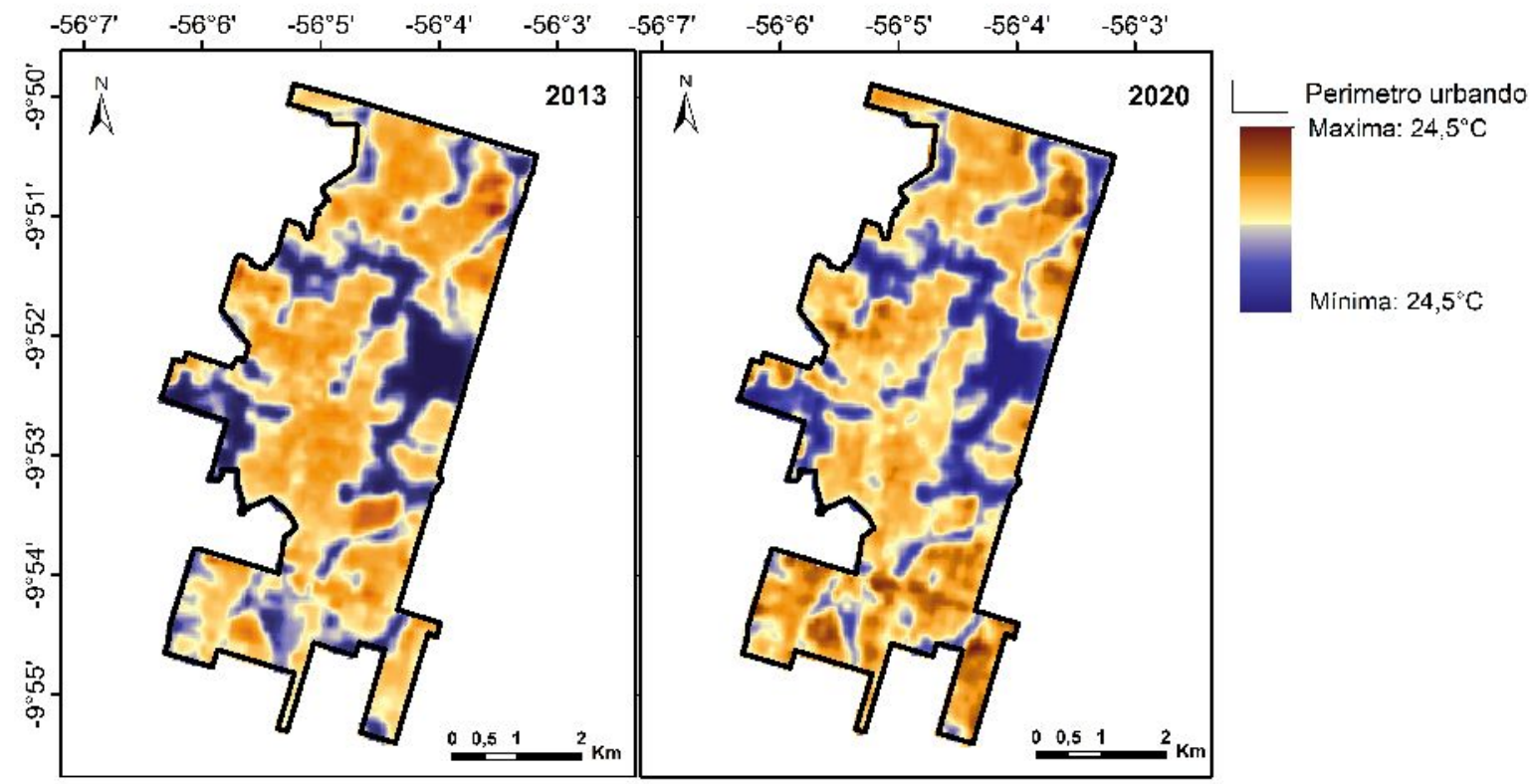

Fonte: próprio autor.

Como um todo, os elevados valores de TS registrados também estão relacionados às imagens terem sido obtidas no mês de junho, que compreende a estação de seca na região. Estudos demonstram que durante a seca há menor presença de nuvens na atmosfera o que facilita a entrada de radiação solar na superfície, assim, sob condições de maior disponibilidade de energia e menor capacidade de conversão dessa energia em calor latente (via evapotranspiração) pelas plantas de pequeno porte (gramíneas) a TS pode aumentar ainda mais e alcançar valores consideráveis (CAIONI et al., 2017; SANTOS et al., 2020).

De modo geral, a temperatura no perímetro urbano de Alta Floresta/MT aumentou $0,8^{\circ} \mathrm{C}$ (Tabela 3 ), isso devido ao também aumento das áreas pavimentadas, visto que essas apresentam maior temperatura, comparada a áreas com cobertura vegetal e massa d' água (GUILHERME et al., 2020). 
TABELA 2. Temperatura da superfície (TS) por classes de uso e cobertura da terra.

\begin{tabular}{|c|c|c|}
\hline Uso e ocupação & TS em 2013 & TS em 20202013 \\
\hline Área pavimentada & $29,94^{\circ} \mathrm{C}$ & $30,10^{\circ} \mathrm{C}$ \\
\hline Cobertura vegetal & $27,52^{\circ} \mathrm{C}$ & $27,95^{\circ} \mathrm{C}$ \\
\hline Massa d' água & $27,50^{\circ} \mathrm{C}$ & $27,42^{\circ} \mathrm{C}$ \\
\hline No geral & $28,8^{\circ} \mathrm{C}$ & $29,6^{\circ} \mathrm{C}$ \\
\hline
\end{tabular}

A TS das aéreas de cobertura vegetal $\left(0,43^{\circ} \mathrm{C}\right)$ aumentou consideravelmente. Esse aumento pode indicar que os remanentes florestais podem estar sofrendo com o aumento das áreas pavimentadas. Isso ocorre porque a umidade presente em locais de alta pressão (remanescentes florestais) tendem a se deslocar para locais de baixa pressão (centro da cidade) (CAIONI et al., 2020). Esse resultado é preocupante, visto que a redução da umidade do ar e o aumento da TS podem levar a alterações na estrutura e composição da floresta, e consequentemente em seus serviços ecossistêmicos prestados.

\section{CONCLUSÕES}

O perímetro urbano apresentou de 2013 a 2020 um considerável aumento das áreas pavimentadas, ao mesmo tempo em que a cobertura vegetal (constituída em grande parte por pastagem exótica) decresceu.

Como um todo, a TS do perímetro urbano aumentou ao longo dos anos, devido principalmente ao surgimento de novas áreas pavimentadas. A TS das áreas de vegetação também demonstraram acréscimos, indicando que a TS advinda das áreas pavimentadas pode estar influenciando a TS dos remanentes florestais.

Levando em consideração que os remanentes florestais tendem a contribuir para a redução da TS no perímetro urbano de Alta Floresta, e que o aumento exacerbado da TS pode comprometer os serviços ecossistêmicos prestados por esses ambientes, sugere-se que novos estudos sejam realizados na área investigada.

\section{REFERÊNCIAS}

AMORIM, M. C. D. C. T. Ilhas de calor urbanas: métodos e técnicas de análise. Revista Brasileira de Climatologia, 2019. Disponível em: < https://revistas.ufpr.br/revistaabclima/article/view/65136/.http://dx.doi.org/10.5380/ab clima.v0i0.65136>. Acessado em: 16 maç. 2021.

ARAUJO, A. L, SILVA, M. T, SILVA, B. B, SANTOS, C. A. C.; AMORIM, M. R. B. Modelagem simplificada para estimativa do balanço de energia à superfície em escala regional (R-SSEB). Revista Brasileira de Meteorologia, v. 32, n. 3, p. 433446 , 2017.

Disponível em: https://www.scielo.br/scielo.php?script=sci_arttext\&pid=S0102-77862017000300433. dx.doi.org/10.1590/0102-77863230010> Acesso em: 17 mar. 2021.

BARBOZA, E. N.; BEZERRA NETO, F. C.; CAIANA, C. R. A. Sensoriamento Remoto aplicado à análise do fenômeno de llhas de Calor Urbano na cidade de Vitória, Espírito Santo. Research, Society and Development, v. 9, n. 6, p. e187963655e187963655, 2020. Disponível em: 
$<$ https://rsdjournal.org/index.php/rsd/article/view/3655/3916. dx.doi.org/10.33448/rsdv9i6.3655> Acesso em: 17 mar. 2021.

BEZERRA, P. E. S.; MORAES, E. T. I.; COSTA SOARES, I. R. Análise da Temperatura de Superfície e do Índice de Vegetação no Município de Belém na Identificação das Ilhas de Calor. Revista Brasileira de Cartografia, v. 70, n. 3, p. 803-818, 2018.

Disponível em: http://www.seer.ufu.br/index.php/revistabrasileiracartografia/article/view/45701. doi.org/10.14393/rbcv70n3-45701>. Acesso em: 15 jun. 2020.

CAIONI, C.; NEVES, S.M.A.S; CAIONI, S.; BONINI, I.; PARENTE, T.L.; SILVA, A.C.S. Dinâmica da temperatura superficial da microbacia Pedra do Índio durante a seca de 2013. Revista Espacios, v.38, n.38, p.9-20, 2017. Disponível em: < https://www.revistaespacios.com/a17v38n38/a17v38n38p09.pdf >. Acesso em: 02 jan. 2021.

CAIONI, C.; CAIONI, S.; SILVA, A.C.S.; PARENTE, T. L.; ARAÚJO, O. S. Análise da distribuição pluviométrica e de ocorrência do fenômeno climático ENOS no município de Alta Floresta/MT. Enciclopédia Biosfera, v.10, n.19, p.2656-2666, 2014. Disponível em: <http://www.conhecer.org.br/enciclop/2014b/MULTID ISCIPLINAR/Analise\%20da\%20distribuicao.pdf>. Acesso em: 02 jan. 2021. Disponível em:

https://www.sciencedirect.com/science/article/abs/pii/0097849396000088.

https://doi.org/10.1016/0097-8493(96)00008-8> Acesso em: 15 jun. 2020.

CAIONI, C.; SILVÉRIO, D. V.; MACEDO, M. N.; COE, M. T.; BRANDO, P. M. Droughts Amplify Differences Between the Energy Balance Components of Amazon Forests and Croplands. Remote Sensing, v. 12, n. 3, p. 525, 2020. Disponível em: < https://www.mdpi.com/2072-4292/12/3/525. 10.3390/rs12030525> Acesso em: 15 jun. 2020.

CÂMARA, G.; SOUZA, R.C.M.; FREITAS, U.M.; GARRIDO, J. SPRING: Integrating remote sensing and GIS by object-oriented data modeling. Computers \& Graphics, v. 20, n. 3, p. 395-403, 1996. Disponível em: < https://www.sciencedirect.com/science/article/abs/pii/0097849396000088. https://doi.org/10.1016/0097-8493(96)00008-8>. Acessado em: 12 Fev. 2021.

GUILHERME, A. P.; BIUDES, M. S.; SANTOS MOTA, D.; MUSIS, C. R. Relação entre tipo de cobertura do solo e temperatura de superfície. Sociedade \& Natureza, v. $32, \quad$ p. 539-550, 2020. Disponível em: < http://www.seer.ufu.br/index.php/sociedadenatureza/article/view/47462. https://doi.org/10.14393/SN-v32-2020-47462>. Acessado em: 16 Mar. 2021.

HIJMANS, R. J.; VAN ETTEN, J.; ETTEN, J.; MATTIUZZI, M. V.; SUMNER, M.; GREENBERG, J. A.; LAMIGUEIRO, O. P.; RACINE, E.B.; SHORTRIDGE, A. raster: Geographic data analysis and modeling. R package version, v.2, p.15, 2014. Disponível em: < https://rdrr.io/cran/raster/>. Acessado em: 16 Mar. 2021. 
IBGE. Instituto Brasileiro de Geografia e Estatística. Confederação Nacional de municípios. 2020. Disponível.em:<https://cidades.ibge.gov.br/brasil/mt/altafloresta/panorama>. Acessado em: 16 maç. 2021.

PESSI, D. D.; EREIO, P. K. B.; ALVES, G. B. M.; MARTARELLO, A. P.; OLIVEIRA, S. M. L. Qualidade da Cobertura Vegetal em Áreas de Preservação Permanente de Nascentes. Anuário do Instituto de Geociências, v.41, n.3, p.270280, 2019. Disponível

em: <http://www.ppegeo.igc.usp.br/index.php/anigeo/article/view/13061. http://dx.doi.org/10.11137/2018_3_270_280> Acesso em: 15 maç. 2021.

PORANGABA, G. F. O.; TEIXEIRA, D. C. F.; AMORIM, M. C. D. C. T. Procedimentos Metodológicos para análise das ilhas de calor em cidades de pequeno e médio porte. Revista Brasileira de Climatologia, v. 21, 2017. Disponível em: $\quad<$ https://revistas.ufpr.br/revistaabclima/article/view/48832. http://dx.doi.org/10.5380/abclima.v21i0.48832> Acesso em: 15 maç. 2021.

PORANGABA, G. F. O.; AMORIM, M. C. D. C. T. Geotecnologias Aplicadasà Análise de Ilhas de Calor de Superfície em Cidades do Interior do Estado de São Paulo. Revista Brasileira de Geografia Física, v.12, n.06, p. 2041-2050, 2019. Disponível.em:<https://periodicos.ufpe.br/revistas/rbgfe/article/viewFile/240229/3435 8. doi.org/10.26848/rbgf.v12.6.p2041-2050>. Acessado em: 16 maç. 2021

SABINO, M.; BOUVIÉ, L.; SILVA JUNIOR, A. A.; MACHADO, N. G.; BIUDES, M. S. Variação espaço-temporal de parâmetros biofísicos e da evapotranspiração em plantios de eucalipto. Ciência e Natura, v. 41, p. 35, 2019. Disponível em: < https://periodicos.ufsm.br/cienciaenatura/article/view/35416/html..http://dx.doi.org/10. 5902/2179460X35416>. Acessado em: 16 maç. 2021.

SANTOS, L. G. F.; FIALHO, E. S. Análise da distribuição da Temperatura de Superfície em relação ao Índice de Vegetação por Diferença Normalizada, em estação seca e chuvosa, no município de Teixeiras-MG. Revista Ponto de vista , v. 9 , n. 3, p. 95-112, 2020. Disponível em: < https://periodicos.ufv.br/RPV/article/view/10805>. Acessado em: 16 maç. 2021.

SILVA, K. B.; COSTAS, P. A. D.; SANTOS, G. P.; GÓES, K. O. Identificação de ilhas de calor: proposta de criação de áreas verdes em média cidade do Sudeste $\mathrm{Da}$ Bahia. Brazilian Journal of Development, v. 6, n. 10, p. 83169-83186, 2020. Disponível.em:<https://www.brazilianjournals.com/index.php/BRJD/article/view/1906 6.. https://doi.org/10.34117/bjdv6n10-667>. Acessado em: 16 maç. 2021.

TEAM, R. CORE. R: A language and environment for statistical computing. $\mathbf{R}$ Foundation for Statistical Computing, Vienna, Austria. 2013. 2014. Disponível em: <URL http://www. R-project. org >. Acessado em: 10 jan. 2021. 